\title{
Plasmonic Color Palettes for Photorealistic Printing with Aluminum Nanostructures
}

\author{
Shawn J. Tan, ${ }^{\dagger}$ Lei Zhang, ${ }^{\dagger}$ Di Zhu, ${ }^{\dagger}$ Xiao Ming Goh, ${ }^{\dagger}$ Ying Min Wang, ${ }^{\dagger}$ Karthik Kumar, ${ }^{\dagger}, \perp$ \\ Cheng-Wei Qiu," and Joel K. W. Yang, ${ }^{*, t}$, \\ ${ }^{\dagger}$ Institute of Materials Research and Engineering, A*STAR, 3 Research Link, Singapore 117602, Singapore \\ ${ }^{\ddagger}$ Department of Electrical and Computer Engineering, National University of Singapore, 4 Engineering Drive 3, Singapore 117583, \\ Singapore \\ ${ }^{\S}$ Singapore University of Technology and Design, 20 Dover Drive, Singapore 138682, Singapore
}

\section{Supporting Information}

ABSTRACT: We introduce the first plasmonic palette utilizing color generation strategies for photorealistic printing with aluminum nanostructures. Our work expands the visible color space through spatially mixing and adjusting the nanoscale spacing of discrete nanostructures. With aluminum as the plasmonic material, we achieved enhanced durability and dramatically reduced materials costs with our nanostructures compared to commonly used plasmonic materials such as gold and silver, as well as size regimes scalable to higherthroughput approaches such as photolithography and nanoimprint lithography. These advances could pave the way toward a new generation of low-cost, high-resolution, plasmonic color printing with direct applications in security tagging, cryptography, and information storage.

KEYWORDS: Nanoplasmonics, aluminum plasmonics, color printing, structural color, electron-beam lithography
Nanoplasmonics involve the collective oscillation of free electrons in nanoscale volumes of metal, enabling the manipulation of light-matter interactions beyond the diffraction limit. ${ }^{1}$ This phenomenon has served as the foundation for a myriad of plasmonic color applications, which includes transmission filters, surface holograms, reflective color printing, and single-particle sensors. ${ }^{2-9}$ In particular, color printing based on the plasmon resonances of metal nanostructures has the advantages of diffraction-limited print resolutions and the simultaneous generation of full colors without the need to deposit different color pigments in a serial manner. Notably, by relying on the plasmon resonances of isolated subwavelength metal nanostructures in the visible light, color printing resolutions have been advanced from $\sim 1000$ dots per inch (dpi) with modern pigment-based color printing to $\sim 100,000$ dpi with plasmonic nanostructures. ${ }^{2}$ Plasmonic color printing technology has significance in data storage and digital imaging as well as security and product-branding applications but is largely limited by practical issues of cost and utility. Thus far, the majority of work on plasmonic color applications, including color printing, employs nanostructures of noble metals such as gold and silver that exhibit colors due to plasmon resonances in the visible spectrum. ${ }^{2,8-13}$ However, despite their potential as a next-generation color-printing technology, there remain key challenges that need to be addressed. For example, the interband transitions of gold limit the rendering of colors at wavelengths less than $\sim 500 \mathrm{~nm}$ and result in a yellow tint. ${ }^{14}$ While silver nanostructures do not present such issues and are able to achieve colors that span the entire visible spectrum, they are extremely susceptible to oxidation and sulphidation, which degrade their colors. These factors, along with the high material costs of both gold and silver, severely hinder the practical applications of these precious metals as materials for plasmonic printing.

Aluminum, on the other hand, is gaining interest as a promising low-cost and sustainable plasmonic material that could extend plasmon resonances into the ultraviolet. ${ }^{14,15}$ From a practical aspect, aluminum is the third most abundant element on earth that is about one-thousandth of the cost of gold or silver. Additionally, its shortcomings in its use as sensors, i.e., its broader resonances and its surface oxidation, become advantages for creating color prints in the visible regime. ${ }^{5,16-18}$ Specifically, aluminum forms a self-limiting impermeable native oxide layer ${ }^{19}$ that affords longer stability over an extended period of time ( $>6$ months) ${ }^{20}$ without requiring additional encapsulation (Figure S2), a vast improvement over the short shelf life of silver as documented in literature. $^{21-23}$ Furthermore, although the low $Q$-factor of

Received: April 20, 2014

Revised: May 28, 2014

Published: June 13, 2014 

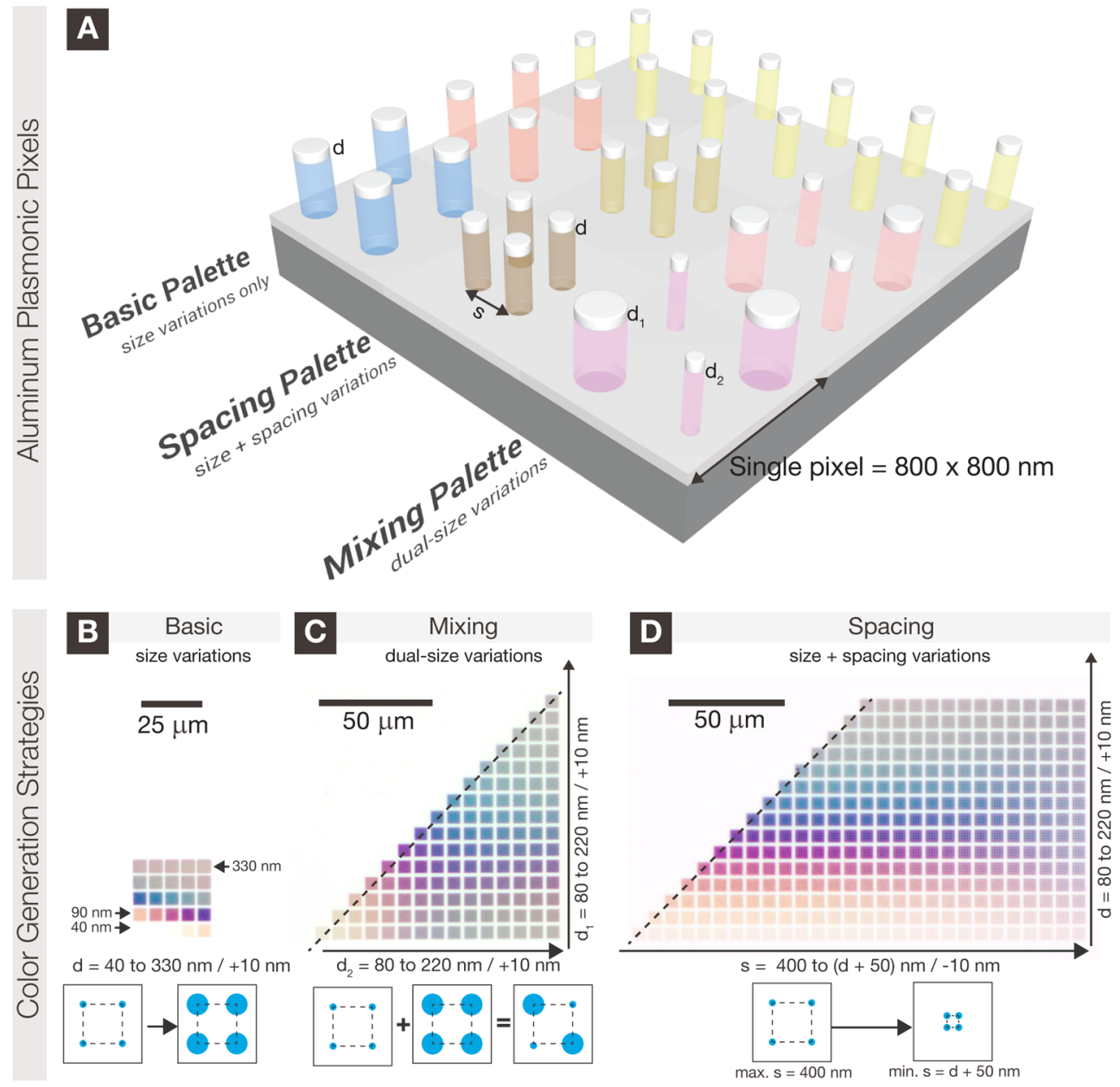

Figure 1. (A) Schematic illustrating the architecture of aluminum plasmonic pixels. Isolated aluminum nanodisks sit atop HSQ nanopillars. The substrate is coated with an aluminum backreflector. Actual experimental images of fabricated color palettes with different layout strategies: (B) basic color palette with only size variations $(d=40$ to $330 \mathrm{~nm})$ at a step size of $10 \mathrm{~nm}$ and a fixed pitch of $400 \mathrm{~nm}$ between nanodisks, (C) mixing color palette with two size variations $\left(d_{1}, d_{2}=80\right.$ to $\left.220 \mathrm{~nm}\right)$ among four nanodisks within an $800 \times 800 \mathrm{~nm}$ pixel, at a step size of $10 \mathrm{~nm}$ and a fixed spacing of $400 \mathrm{~nm}$ between nanodisks, and (D) spacing color palette with both size variations $(d=80$ to $220 \mathrm{~nm})$ and spacing variations $(s=d+50$ to $400 \mathrm{~nm}$ ) among four nanodisks within an $800 \times 800 \mathrm{~nm}$ pixel, at a step size of 10 and $20 \mathrm{~nm}$, respectively. Optical images were normalized to a white background.

aluminum in the visible regime might be a drawback for many applications, ${ }^{14,24}$ it is a useful feature for plasmonic color printing: The lower spectral sensitivity requires larger structural differences to achieve distinct colors, which increases the tolerance to critical-dimension control, making it advantageous for mass production via high-throughput lithographic processes. Thus, aluminum has quickly emerged as an attractive material for exploring plasmon-based color in both transmission and reflection modes. ${ }^{5,16-18}$

Given the appeal of aluminum for plasmonic color printing, it is imperative to develop a generalized account of design strategies for color generation with "plasmonic pixels". Traditional pigment-based printing achieves a full spectrum of colors through mixing and toning strategies involving primary colors; for example color mixing is achieved by overlaying different colors, while color toning is achieved by adjusting the size and density of ink droplets. However, such strategies are not directly applicable in plasmonic color printing, in which the scattered colors defined by the resonant wavelengths are in turn determined by the size of the metal nanostructures. Furthermore, in the case of pigment-based color printing, various color systems such as RGB (red-green- blue), CMYK (cyan-magenta-yellow-black), and HSL (hue, saturation, lightness) have been established to describe generated colors from their respective primary color parameters. However, such color systems have not been established for plasmonic-based colors. Despite the well-established phenomenon of color in pixels consisting of single plasmonic nanostructures, there is yet a focused study on achieving a broad color palette using color generation strategies with multiple nanostructures within a single pixel. In the case of aluminum color pixels, despite its practical advantages for plasmonic color printing, color generation and mixing strategies are ultimately necessary to compensate for the low Q-factor that reduces the purity of the colors.

Here, we present a study for color generation and the design principle of plasmonic color pixels. Specifically, with aluminum as the plasmonic material, we expand the range of printable plasmonic colors from $\sim 15$ colors to more than 300 colors by employing newly defined color generation strategies as shown in Figure 1. With each single pixel comprising four nanodisks, we achieved color creation by positioning different-sized disks within a pixel (i.e., the mixing palette) and further fine-tuned colors by adjusting the spacing between the disks (i.e., the 

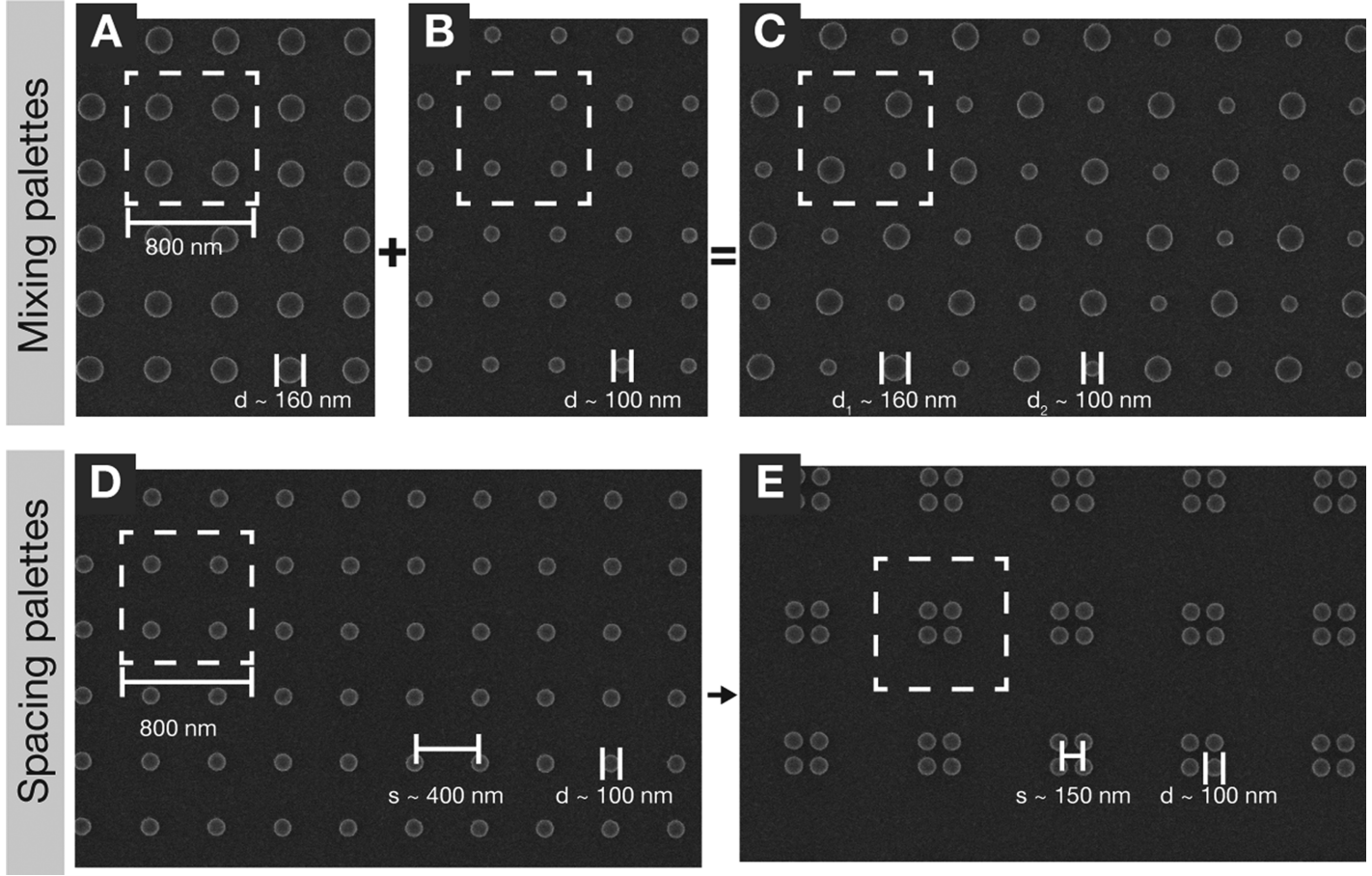

Figure 2. SEM characterization of representative arrays within mixing and spacing palettes reveal the nanoscale layout within the $800 \mathrm{~nm} x 800 \mathrm{~nm}$ pixel area specified by dotted lines. Mixing individual nanodisk features with diameter (A) $d=160$ and (B) $d=100 \mathrm{~nm}$ result in a mixed palette (C) with two nanodisks of diameters $d_{1}=160$ and $d_{2}=100 \mathrm{~nm}$ within a single plasmonic pixel. Spacing palettes are achieved by varying the center-tocenter spacing between nanodisks of the same diameters within the plasmonic pixel, such as from (D) $s=400 \mathrm{~nm}, d=100 \mathrm{~nm}$ to (E) $s=150 \mathrm{~nm}, d$ $=100 \mathrm{~nm}$.

spacing palette). In addition, we demonstrate that aluminum is an excellent material for plasmonic color printing due to its durability and color generation with size tolerances that reduce complexity for mass production via photolithography or nanoimprinting techniques. These key advances bring us a step closer toward full color competency in plasmonic color printing and could pave the way for a new generation of lowcost yet high-resolution, nanometallic-based color prints with direct applications in security tagging, cryptography, and data storage. $^{25-27}$

To begin, we fabricated $95 \mathrm{~nm}$-tall nanopillars of varying diameters on a silicon substrate using electron-beam lithography (EBL) patterning of the negative-tone hydrogen silsequioxane (HSQ) resist. ${ }^{2}$ The EBL-patterned HSQ was developed in the "salty developer", i.e., an aqueous solution of $4 \% \mathrm{NaCl}$ and $1 \% \mathrm{NaOH}$ for $1 \mathrm{~min}^{28}$ At this point, the bare nanopillars do not yet exhibit plasmonic colors. The colors are revealed only after depositing $20 \mathrm{~nm}$-thick aluminum onto the nanopillar patterns in an electron-beam evaporator (see Materials and Methods in SI for more details). Due to the directional deposition of the metal, the final plasmonic nanoprints are composed of aluminum nanodisks atop HSQ nanopillars and an aluminum back-reflector film in between the pillars (Figure 1A). As aluminum acts as an excellent backreflector, with a reflectance of over $90 \%$ at visible light, near-IR, and IR wavelengths, ${ }^{29}$ it provides a neutral "white" background for color nanoprints under normal incidence viewing rather than a tinted background from metals such as gold or copper.

Basic palettes of primary plasmonic colors were fabricated by patterning multiple square arrays of aluminum nanodisks at a pitch of $400 \mathrm{~nm}$. Each $4 \mu \mathrm{m}$ square array consists of disks of uniform diameters. The diameters of disks varied from array to array from 40 to $330 \mathrm{~nm}$ at a step size of $10 \mathrm{~nm}$, with the smallest disks on the bottom right array and the largest on the top right (Figure 1B). A total of $\sim 15$ distinct colors ranging from yellow, orange, red, purple, and blue to green were achieved for aluminum nanodisks within a limited range of 80$220 \mathrm{~nm}$ in diameter. Aluminum nanodisks with diameters smaller than $80 \mathrm{~nm}$ and larger than $220 \mathrm{~nm}$ did not generate distinct colors due to the lack of resonances in the visible range. As the suitable sizes of aluminum nanostructures for color generation are above $80 \mathrm{~nm}$, these structures are sufficiently large and amenable for mass production using modern photolithography and nanoimprint techniques, once master reticles are created using maskless methods such as EBL and focused ion-beam (FIB) milling. For the same reason, we chose a technologically attainable step size that was no smaller than $10 \mathrm{~nm}$ for the aluminum color palettes. In comparison, the optimal parameters for color palettes with gold/silver nanostructures required smaller diameters $(50-140 \mathrm{~nm})$ and gaps (as small as $30 \mathrm{~nm}$ ), which would place more stringent requirements for high-throughput pattern replication methods. ${ }^{2}$ Nevertheless, despite the advantages of aluminum nanostructures for color printing, the basic palette of primary plasmonic colors is deficient in color variety (with only $\sim 15$ distinct colors as shown in Figure 1B). Thus, in order to achieve photorealistic color printing with more colors and variations in tones, new strategies for color generation must be developed.

To this end, we developed plasmonic color "mixing" and "spacing" palettes based on the basic palette, which are analogous to the strategies of mixing and toning used in inkbased color printing. For each layout, we defined each "plasmonic pixel" with an $800 \times 800 \mathrm{~nm}$ pixel area to comprise of four aluminum nanodisks. In each pixel of the "mixing" palette, four aluminum nanodisks were positioned at a fixed 


\section{A Experimental}
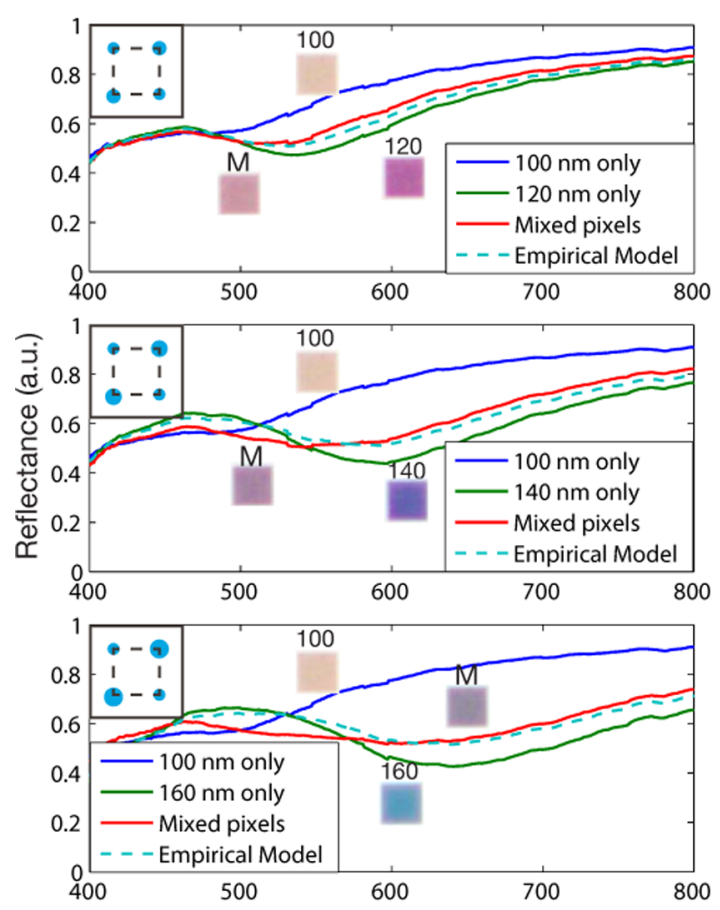

Wavelength $(\mathrm{nm})$

\section{E-field in x-z plane @ 466 nm}
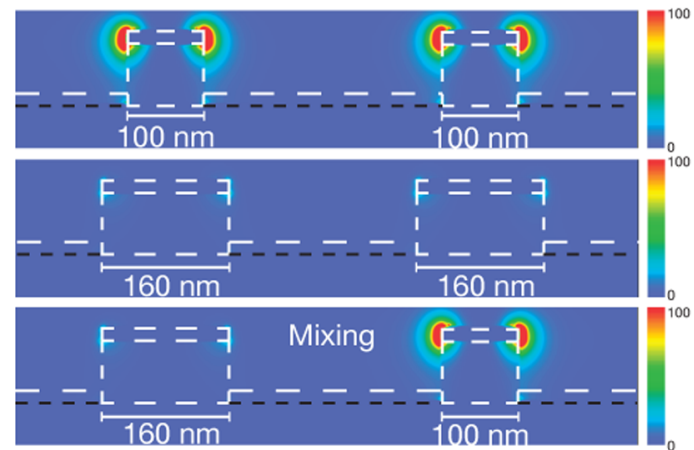

\section{B FDTD Simulations}
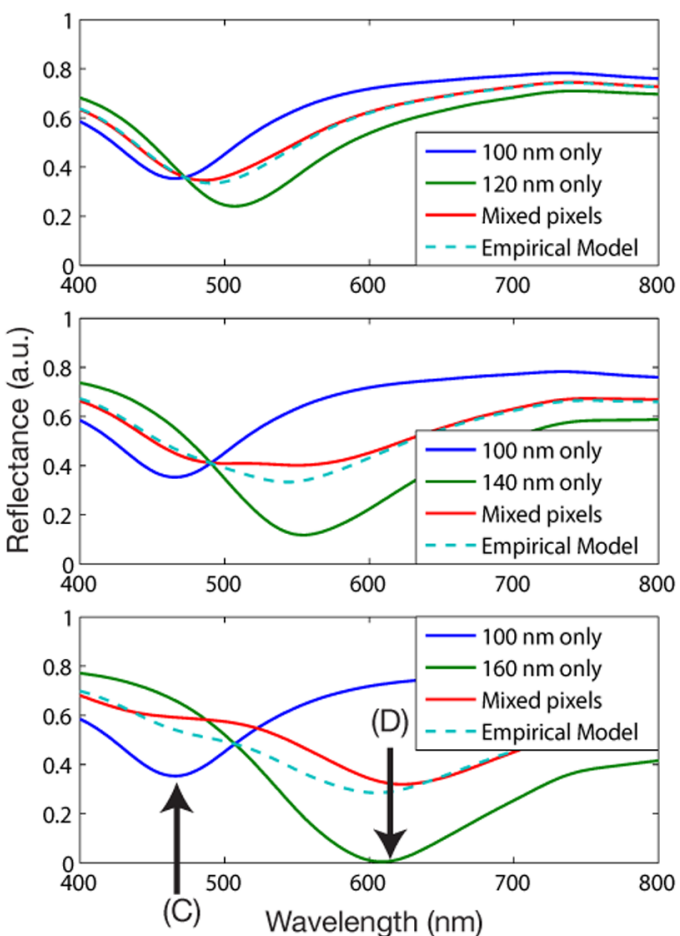

D E-field in x-z plane @ 624 nm

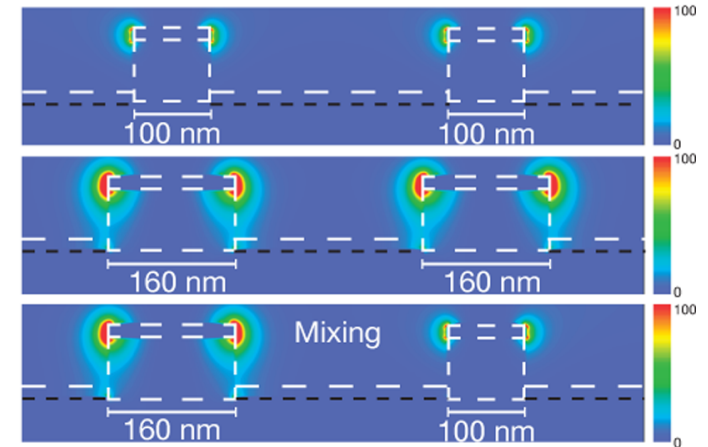

Figure 3. (A) Experimental spectra of mixing palettes containing two different diameters of nanodisks, $d_{1}$ and $d_{2}$, within the plasmonic pixel. Each subplot shows the experimental spectrum of $d_{1}=d_{2}=100 \mathrm{~nm}$ (blue lines), $d_{1}=d_{2}=120,140$, or $160 \mathrm{~nm}$ (green lines), and the mixed palettes of $d_{1}$ $=100 \mathrm{~nm} ; d_{2}=120,140$, or $160 \mathrm{~nm}$ (red lines). The teal dotted line is obtained empirically through the weighted average of $d_{1}$ and $d_{2}$ spectra. Optical images of the corresponding arrays in the mixing palette are included for comparison. (B) Simulations corresponding to the experimental spectra shown in (A). From top to bottom, electric field in the $x-z$ plane for basic palettes of 100 and $160 \mathrm{~nm}$ as well as a mixed palette of $100 \times$ $160 \mathrm{~nm}$ nanodisks at incident wavelengths of (C) 466 and (D) $624 \mathrm{~nm}$, corresponding to dips revealed in simulation results. White dotted lines outline the structural features of nanodisks, nanopillars, and backreflector layer. Black dotted lines outline the substrate layer.

center-to-center pitch of $400 \mathrm{~nm}$, with alternating diameters of $d_{1}$ and $d_{2}$ ranging from 80 to $220 \mathrm{~nm}$ in steps of $10 \mathrm{~nm}$ (Figure 1C). The diagonal edge of the color palette cuts across the color arrays with equal diameters $\left(d_{1}=d_{2}\right)$, corresponding to the basic palette in Figure 1B. The remaining colors were achieved by mixing disks of different diameters within a single pixel, allowing a full array of more than a hundred colors to be derived from the primary plasmonic colors. In each pixel of the "spacing" palette, four aluminum nanodisks with diameters, $d$, ranging from 80 to $220 \mathrm{~nm}$ in steps of $10 \mathrm{~nm}$, were positioned at a varying center-to-center distance of $(d+50)$ to $400 \mathrm{~nm}$ in steps of $10 \mathrm{~nm}$ (Figure 1D). Again, the diagonal edge corresponds to the basic palette in Figure 1B. With the spacing palette, about 200 new colors were obtained with the diameter ranging from 100 to $180 \mathrm{~nm}$. Notably, the colors achieved with the spacing palette are subtle variations of the primary plasmonic colors yet distinct from those generated through the mixing palette. In addition, these palettes were exposed to ambient conditions for more than 7 months as a test of their durability (Figure S2) and showed no signs of deterioration. In comparison, silver nanodisks exhibited the well-known signs of deterioration within a week due to the susceptibility of silver to oxidation and sulphidation.

Figure 2 shows scanning electron micrographs (SEM) of the metallic nanostructures. Excellent agreement in size between the designed layout and the fabricated nanostructures was observed in both the mixing and spacing palettes. These SEM images indicate that the structures were patterned with 
sufficiently large separations such that the mixing of different sized disks did not result in any observable electron-beam proximity effects. ${ }^{30}$ Therefore, the potential size variations in the EBL exposure, which may complicate our analysis and deteriorate the final performance, were effectively suppressed. Measurement of collapsed aluminum nanodisks on HSQ nanopillars revealed a total height of $115 \mathrm{~nm}$, composed of $20 \mathrm{~nm}$-tall aluminum nanodisks on $95 \mathrm{~nm}$-tall HSQ nanopillars (Figure S3).

To quantify the colors of the mixed pixels and devise an empirical model, we measured the reflection spectra for the basic color pixels $(d=100,120,140$, and $160 \mathrm{~nm})$ and their corresponding mixed pixels $\left(d_{1}=100 \mathrm{~nm}, d_{2}=120,140\right.$, and $160 \mathrm{~nm})$, as shown in Figure 3A. As the diameters of the nanodisks increased from 100 to $160 \mathrm{~nm}$ in the basic color pixels, we observed a corresponding red-shift of the resonance due to an increase in dipole moment, which is consistent with the trend previously observed for gold/silver nanodisks. ${ }^{2,31}$ The colors of the pixels observed under an optical microscope were also included alongside the reflection spectra, illustrating that hybrid nanodisks of different diameters within a single pixel indeed result in new colors (Figure 3A,B). Intuitively, their resultant spectra (i.e., secondary colors) appear to be a function of the spectra of their basic-color palettes (i.e., primary colors). To obtain a plausible design recipe, a phenomenological expression was developed to estimate the mixed-pixel spectrum using a diameter-weighted average of the individual spectra:

$$
R(\lambda)=\left(\frac{d_{1}}{d_{1}+d_{2}}\right) R_{1}(\lambda)+\left(\frac{d_{2}}{d_{1}+d_{2}}\right) R_{2}(\lambda)
$$

where $R$ is the resulting reflectance of a single pixel with mixed nanodisk diameters at a given wavelength $\lambda, d_{1}$ and $d_{2}$ are the respective diameters of nanodisks within the plasmonic pixel, and $R_{1}$ or $R_{2}$ are the reflectance spectra of pixels comprising nanodisks with diameters $d_{1}$ or $d_{2}$. This estimate agrees with the experimentally observed spectra for the mixed plasmonic pixels, as shown in Figure 3A.

Given that we could not achieve perfectly matched mixed spectra with the linear combination of individual spectra, it suggests that plasmonic color mixing in our pixel design may be more complex and involves the consideration of local resonances of the combined disk configurations. We notice that the mixed spectra match the empirical spectra of larger disks at longer wavelengths and the smaller disks for the shorter wavelengths. This effect suggests that the intensity of the mixed spectra is predominantly determined by the resonances of individual disks in the pixel, i.e., minimal interaction between adjacent nanopillars was involved in the color generation. Consistent with single pixel characterization, the individual nanodisks within each pixel could be resolved and manifested as different colors (Figure S4). The colors due to slight variations in the shape and size of individual disks can be seen in Figure S4, where structures were fabricated with rough edges to test the tolerance of the process to patterning errors.

To further reveal the underlying mechanism, finite-difference time-domain (FDTD) simulations were performed to calculate the reflectance spectra and the electric near-field distributions of the proposed plasmonic pixels. As shown in Figure 3B, the simulated reflectance spectra of the individual and mixed pixels were consistent with the experimental measurements. The redshift of the experimental spectra relative to the simulated results may be attributed to the effects arising from the native aluminum oxide layer, ${ }^{24}$ structural variations in fabrication, and the curved side-wall profiles of our structures (not taken into consideration in the model). The empirical model in eq 1 for predicting the spectra of the mixed plasmonic pixel works well in the simulation results. Field plots of the plasmonic pixels in the vertical plane that bisects the disks are shown in Figure 3C,D. These plots, simulated at the individual resonances for the different-sized disks, illustrate that coupling of the plasmon modes is negligible. Instead, the resonances of individual nanodisks are preserved within the mixed plasmonic pixel and occur independently of each other. This color independence of individual nanodisks in our layout enables an easy-to-access color system to engineer plasmonic colors. While the mixing palettes enable generation of distinct secondary colors by incorporating nanodisks of different sizes within one pixel, the spacing palettes enable a finer tuning of the colors. The effect of changing the spacing is characterized in Figure 1D and further verified in the spectra shown in Figure S6. Notably, with decreasing interdisk spacing, we observed a broadening and flattening of the plasmon resonance dip and a corresponding decrease in the spectral purity, which leads to subtle changes in the tone of the color reflected (see Figure S6). The peaks observed in the experimental spectra but not in the simulation are likely to be artifacts due to the diffraction orders inadvertently collected by the microspectrophotometer.

The mixing and spacing palettes discussed above have generated more than 300 unique colors, a significant improvement over the original basic palette with only $\sim 15$ colors. To demonstrate the versatility of the proposed approach, we expanded the design of our mixing and spacing palettes to nanodisks spaced at a pitch of $200 \mathrm{~nm}$ in addition to the 400 $\mathrm{nm}$ pitch arrays (Figure S7). The extended palettes occupy a larger region of the CIE 1391 color space (Figure S8) that is commonly used to describe perceived colors. Interestingly, our mixing strategy led to the generation of new colors beyond the region enclosed by the basic plasmonic colors, unlike the case for pigments and chromophores for which the primary colors define the boundaries of the color map.

To highlight the range of colors that could be generated, we printed Claude Monet's painting of Impression, Sunrise in which subtle color variations and tones were utilized. The digital image was input (Figure 4A) into a MATLAB script that searched for the closest RGB color match for the individual pixels to the various color palettes and generated a layout of nanodisks with different sizes and spacing. Using only the basic palette of primary plasmonic colors, the resulting print was unable to capture the subtle variations in color and or the details in the brush strokes (Figure 4B). On the contrary, we successfully printed a "plasmonic painting" based on the extended palettes, with fidelity down to the brush strokes characteristic of Monet's painting (Figure 4C,D), while achieving a remarkable 1000 -fold reduction in size, from an $\sim 80 \mathrm{~cm}$ painting in real-life down to a $\sim 300 \mu \mathrm{m}$ microimage.

In summary, we demonstrated for the first time, the utility of aluminum nanostructures for ultrahigh definition plasmonic color printing. Aluminum becomes an attractive material for plasmonic color printing owing to its neutral tint, durability, resonance insensitivity to size, high reflectance in the visible regime, and global abundance, therefore low cost. Unlike gold and silver, the colors in the visible wavelength regime for aluminum are manifested by nanodisks with feature sizes accessible for mass production. With aluminum nanodisks as our model system, we developed and validated a framework for 

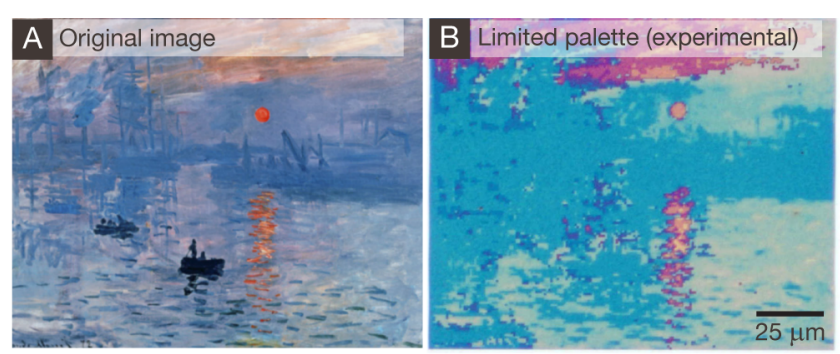

C Expanded palette (experimental)

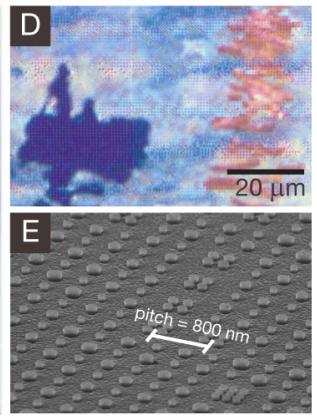

Figure 4. Reproduction of Monet's Impression, Sunrise using the expanded palette by color toning and mixing strategies. (A) Original input image adapted with permission from Musee Marmottan Monet, Paris, France / Giraudon / Bridgeman Images. (B) Reproduction using only the limited palette of "primary plasmonic colors" falls short of the original image. (C) Realistic reproduction of the artwork using an expanded palette of colors, allowing for the subtle variations in tone and color in the original to the replicated. (D) Higher magnification image of dotted box in (C) highlighting the brush strokes that are resolved in the plasmonic painting. (E) Tilted SEM image of pixels showing how the colors observed in the plasmonic painting are the manifestation of a predefined structural layout.

color generation in the context of plasmonic colors that serve to establish a plasmonic color system. Experimental results and simulations unanimously show the color independence of primary plasmonic colors, which allows direct mixing of plasmonic colors within a single pixel. The colors could also be fine-tuned by adjusting the spacing between nanodisks within a plasmonic pixel. With these two strategies as a basis for color generation, we successfully expanded the color palette from $\sim 15$ basic colors to more than 300 unique colors, enabling the printing of photorealistic images.

\section{ASSOCIATED CONTENT}

\section{S Supporting Information}

Additional information including details on methods and materials, simulations, single-pixel resolution, tolerance to edge roughness, measurements of height of structures, color palettes using a smaller pitch of $200 \mathrm{~nm}$, and color space analysis. This material is available free of charge via the Internet at http://pubs.acs.org.

\section{AUTHOR INFORMATION}

\section{Corresponding Author}

*E-mail: joel_yang@sutd.edu.sg.

\section{Present Address}

${ }^{\perp}$ Biomedical Research Council, A*STAR, 20 Biopolis Way, Singapore 138668

\section{Author Contributions}

The manuscript was written through contributions of all authors. All authors have given approval to the final version of the manuscript.

\section{Notes}

The authors declare no competing financial interest.

\section{ACKNOWLEDGMENTS}

The authors would like to acknowledge the funding support from the Institute of Materials Research and Engineering, Agency for Science, Technology and Research (A*STAR) and the National Research Foundation (grant award no. NRF CRP 8201107). L.Z. and C.-W.Q acknowledge the grant R-263-000A45-112 from National University of Singapore. The authors also thank J. Deng, H. Liu, and V. S. F. Lim from the Institute of Materials Research and Engineering Singapore for technical assistance and useful discussions.

\section{ABBREVIATIONS}

SERS, surface-enhanced Raman scattering; EBL, electron beam lithography; HSQ hydrogen silsesquioxane; MIBK, methyl isobutyl ketone; SEM, scanning electron microscopy; FDTD, finite-difference time domain; TFSF, total field scattered field; CIE, Commission Internationale de l'Eclairage

\section{REFERENCES}

(1) Tan, S. J.; Campolongo, M. J.; Luo, D.; Cheng, W. Nat. Nanotechnol. 2011, 6, 268-276.

(2) Kumar, K.; Duan, H.; Hegde, R. S.; Koh, S. C. W.; Wei, J. N.; Yang, J. K. W. Nat. Nanotechnol. 2012, 7, 557-561.

(3) Liu, N.; Tang, M. L.; Hentschel, M.; Giessen, H.; Alivisatos, A. P. Nat. Mater. 2011, 10, 631-636.

(4) Zeng, B.; Gao, Y.; Bartoli, F. J. Sci. Rep. 2013, 3, 2840.

(5) Yokogawa, S.; Burgos, S. P.; Atwater, H. A. Nano Lett. 2012, 12, 4349-4354.

(6) Ozaki, M.; Kato, J.; Kawata, S. Science 2011, 332, 218-220.

(7) Fischer, H.; Martin, O. J. F. Opt. Express 2008, 16, 9144.

(8) Wu, Y.-K. R.; Hollowell, A. E.; Zhang, C.; Guo, L. J. Sci. Rep. 2013, 3, 1194.

(9) Tan, S. J.; Goh, X. M.; Wang, Y. M.; Yang, J. K. W.; Teng, J. J. Mol. Eng. Mater. 2014, in press.

(10) Lochbihler, H. Opt. Express 2009, 17, 12189.

(11) Park, G.; Lee, C.; Seo, D.; Song, H. Langmuir 2012, 28, 90039009.

(12) Roberts, A. S.; Pors, A.; Albrektsen, O.; Bozhevolnyi, S. I. Nano Lett. 2014, 14, 783-787.

(13) Pietrobon, B.; McEachran, M.; Kitaev, V. ACS Nano 2009, 3, $21-26$.

(14) West, P. R.; Ishii, S.; Naik, G. V.; Emani, N. K.; Shalaev, V. M.; Boltasseva, A. Laser Photon. Rev. 2010, 4, 795-808.

(15) Knight, M. W.; Liu, L.; Wang, Y.; Brown, L.; Mukherjee, S.; King, N. S.; Everitt, H. O.; Nordlander, P.; Halas, N. J. Nano Lett. 2012, 12, 6000-6004.

(16) Chen, Q.; Cumming, D. R. S. Opt. Express 2010, 18, 1405614062.

(17) Ellenbogen, T.; Seo, K.; Crozier, K. B. Nano Lett. 2012, 12, 1026-1031.

(18) Zhang, J.; Ou, J.-Y.; Papasimakis, N.; Chen, Y.; Macdonald, K. F.; Zheludev, N. I. Opt. Express 2011, 19, 23279-23285.

(19) Kulkarni, V.; Prodan, E.; Nordlander, P. Nano Lett. 2013, 13, 5873-5879.

(20) Jeurgens, L.; Sloof, W.; Tichelaar, F.; Mittemeijer, E. Phys. Rev. B 2000, 62, 4707-4719.

(21) Bennett, H. E. J. Appl. Phys. 1969, 40, 3351.

(22) Huang, T.; Nancy Xu, X.-H. J. Mater. Chem. 2010, 20, 98679876.

(23) Qi, H.; Alexson, D.; Glembocki, O.; Prokes, S. M. Nanotechnology 2010, 21, 215706.

(24) Langhammer, C.; Schwind, M.; Kasemo, B.; Zorić, I. Nano Lett. 2008, 8, 1461-1471. 
(25) Cui, Y.; Hegde, R. S.; Phang, I. Y.; Lee, H. K.; Ling, X. Y. Nanoscale 2014, 6, 282-288.

(26) Perez-Juste, J.; Mulvaney, P.; Liz-Marzan, L. M. Int. J. Nanotechnol. 2007, 4, 215-225.

(27) Mansuripur, M.; Zakharian, A. R.; Lesuffleur, A.; Oh, S.-H.; Jones, R. J.; Lindquist, N. C.; Im, H.; Kobyakov, A.; Moloney, J. V. Opt. Express 2009, 17, 14001.

(28) Yang, J. K. W.; Berggren, K. K. J. Vac. Sci. Technol., B: Microelectron. Nanometer Struct. 2007, 25, 2025.

(29) Ehrenreich, H.; Philipp, H.; Segall, B. Phys. Rev. 1963, 132, $1918-1928$.

(30) Yang, J. K. W.; Dauler, E.; Ferri, A.; Pearlman, A.; Verevkin, A.; Gol'tsman, G.; Voronov, B.; Sobolewski, R.; Keicher, W. E.; Berggren, K. K. Appl. Supercond. IEEE Trans. 2005, 15, 626-630.

(31) Temple, T. L.; Bagnall, D. M. J. Appl. Phys. 2011, 109, 084343. 